\title{
How the first stars shaped the faintest gas-dominated dwarf galaxies
}

\author{
Robbert Verbeke, Bert Vandenbroucke and Sven De Rijcke \\ Sterrenkundig Observatorium, Ghent University \\ Krijgslaan 281, S9, 9000 Gent, Belgium \\ email: robbert.verbeke@ugent.be
}

\begin{abstract}
Cosmological simulations predict that dark matter halos with circular velocities lower than $30 \mathrm{~km} / \mathrm{s}$ should have lost most of their neutral gas by heating of the ultra-violet background. This is in stark contrast with gas-rich galaxies such as e.g. Leo T, Leo P and Pisces A, which all have circular velocities of $\sim 15 \mathrm{~km} / \mathrm{s}$ (Ryan-Weber et al. 2008, Bernstein-Cooper et al. 2014, Tollerud et al. 2015). We show that when we include feedback from the first stars into our models, simulated dwarfs have very different properties at redshift 0 than when this form of feedback is not included. Including this Population-III feedback leads to galaxies that lie on the baryonic Tully-Fisher relation over the entire mass range of star forming dwarf galaxies, as well as reproducing a broad range of other observational properties.
\end{abstract}

Keywords. dwarf galaxies, first stars, galaxy formation, galaxy evolugtion.

\section{Introduction}

It is thought that the first generation of stars (Population-III or Pop-III stars), that formed out of pristine gas in the early universe, would have a very top-heavy initial mass function (Susa et al. 2014). For a given stellar population, such a Susa-IMF would result in $\sim 4$ times more feedback from supernovae type II and 40 times more in the form of ionizing UV radiation. Here, we use computer simulations to investigate the influence this Pop-III feedback has on the evolution of dwarf galaxies and whether it can alleviate tension between cosmological simulations and observed faint dwarf galaxies.

\section{Baryonic Tully-Fisher relation}

The baryonic Tully-Fisher relation links the total baryonic mass of a galaxy to its circular velocity (e.g. McGaugh 2012), or equivalently, its dynamical mass. Galaxy simulations generally have a hard time reproducing this relation down to the faintest galaxies. Their stellar mass is usually too high for their circular velocity, because these simulations form too many stars at high redshift. When we include feedback from the first generation of stars, the star formation rate at high redshift is significantly reduced. In Figure 1, we show that these simulations (stars) agree with the observed baryonic Tully-Fisher relation over the entire range of star forming dwarf galaxies. The simulations without Population-III feedback (triangles) on the other hands have stellar masses that are too high for their circular velocities.

\section{Other properties}

The process of galaxy formation is very challenging to model and the observable properties of simulated galaxies will strongly depend on the chosen set of parameters. While 


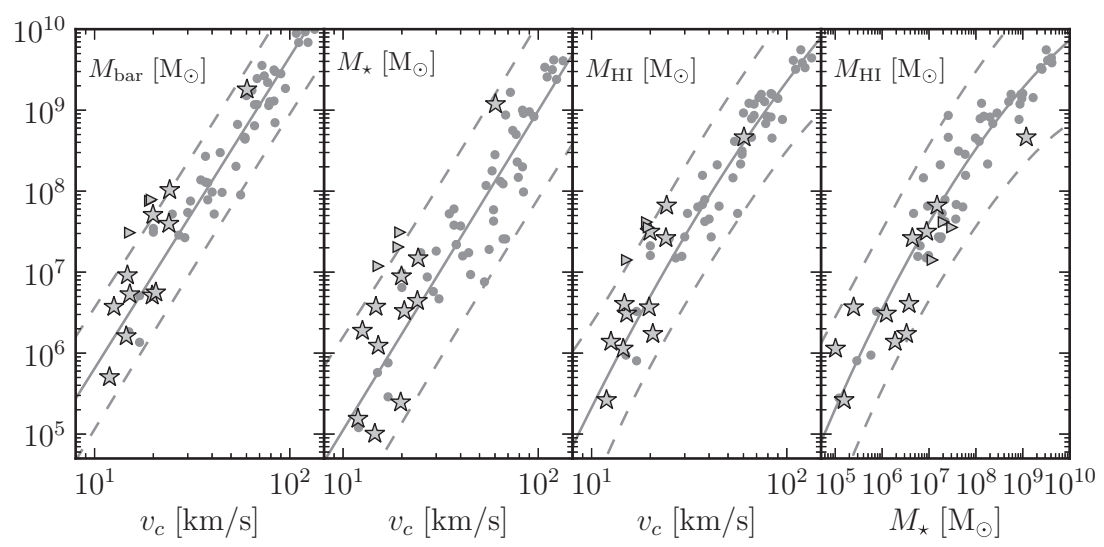

Figure 1. Baryonic Tully-Fischer relations. Baryonic mass (a), stellar mass (b), neutral gas mass (c) versus circular velocity and neutral gas versus stellar mass (d), compared to observations (grey circles; McGaugh 2012). Stars indicate simulations including Pop-III feedback, triangles are simulations without Pop-III feedback.

parameters may be tuned to get one or several galaxy properties in agreement with observations, reproducing a broad range of them is not a trivial task. However, failure to reproduce one or more observed galaxy properties may indicate that an important physical process is missing in the models. This missing ingredient might in turn strongly affect the previous results. We thus stress the importance of comparing a broad range of galaxy properties to observations. We have compared the star formation histories, metallicity, metallicity distribution functions, $M_{\star}-M_{\text {halo-relation, the HI-distribution, }}$ optical colours, the half-light radius, central surface brightness, stellar velocity dispersion, star formation rate, the Kennicutt-Schmidt relation and the total mass within the half-light radius and $300 \mathrm{pc}$, and found good agreement with observations. A more in depth discussion of these properties can be found in Verbeke et al. (submitted).

\section{Conclusions}

We have shown that when including feedback from the first stars in computer simulations, we obtain dwarf galaxies that agree very well with real dwarfs. Since this is generally not true for simulations without pop III stars, we conclude that the first stars that formed in the universe had an important impact on the formation of the faintest dwarf galaxies, which can still be seen today.

\section{References}

Bernstein-Cooper, E. Z., Cannon, J. M., Elson, E. C., et al. 2014, AJ, 148, 35

McGaugh, S. S. 2012, AJ, 143, 40

Ryan-Weber, E. V., Begum, A., Oosterloo, T., et al. 2008, MNRAS, 384, 535

Susa, H., Hasegawa, K., \& Tominaga, N. 2014, ApJ, 792, 32

Tollerud, E. J., Geha, M. C., Grcevich, J., Putman, M. E., \& Stern, D. 2015, ApJL, 798, L21

Verbeke, R., Vandenbroucke, B., \& De Rijcke, S., Submitted to ApJ 